\title{
Barycentric Interpolation Collocation Method for the Nonlinear Korteweg-de Vries Burgers' Equation
}

\author{
Feifei Liu ${ }^{1}$, Wei Liu ${ }^{1}$, Yulan Wang ${ }^{1} \&$ Zhiyuan $\mathrm{Li}^{1}$ \\ ${ }^{1}$ College of Science, Inner Mongolia University of Technology, Hohhot, China \\ Correspondence: Wei Liu, College of Science, Inner Mongolia University of Technology, Hohhot, China. E-mail: \\ zhlnei@163.com
}

Received: May 21, 2017

Accepted: June 11, 2017

Online Published: July 10, 2017

doi:10.5539/mas.v11n8p118

URL: https://doi.org/10.5539/mas.v11n8p118

\begin{abstract}
The Korteweg-de Vries-Burgers (KdVB) equation plays an important role in both physics and applied mathematics, and it had been solved by many methods. In order to obtain more accurate numerical solutions, we introduce a barycentric interpolation collocation method (BICM) for solving the equation and obtain good results. Several numerical examples are selected to verify the high accuracy of the present method.
\end{abstract}

Keywords: Korteweg-de Vries-Burgers equation, barycentric interpolation collocation method, direct linearized iterative, partial differential matrix

\section{Introduction}

The KdVB equation was derived by Su and Gardner for a wide class of nonlinear systems in the weak nonlinearity and long wavelength approximations because it contains both damping and dispersion [1]. The KdVB equation has the following form:

$$
u_{t}+\varepsilon u u_{x}-v u_{x x}+\mu u_{x x x}=0, a<x<b, t>0 .
$$

Where $\varepsilon, \nu$, and $\mu$ are positive parameters.

When $\mu=0$, Eq.(1) is reduced to the Burgers' equation£

$$
u_{t}+\varepsilon u u_{x}-v u_{x x}=0 . a<x<b, t>0 .
$$

When $v=0$, Eq.(1) is reduced to the Korteweg-de Vries equation (KdV for short):

$$
u_{t}+\varepsilon u u_{x}+\mu u_{x x x}=0, a<x<b, t>0 .
$$

There are many numerical methods for the KdV equation [2-4]. The Burgers' equation plays an important role in many fields of applied science, and there are numerous numerical methods for the solution of Burgers' equation in the literature [5-8]. In this paper, the BICM is proposed for solving the KdVB.

The framework of this article is as follows. In Section 2, we introduce BICM in detail. Several numerical examples and some relevant figures and tables are provided in Section 3. Discussion are given in Section 4.

\section{Barycentric Interpolation Collocation Method}

\subsection{Direct Linearized Iterative Method}

We divided a nonlinear $\operatorname{KdVB}$ equation $\mathcal{D} u(x, t)=f(x, t)$ into two parts, which are linear term and nonlinear term:

$$
\mathcal{L} u(x, t)+\mathcal{N} u(x, t)=f(x, t)
$$

where $\mathcal{L}$ and $\mathcal{N}$ are linear and nonlinear differential operators, respectively.

Assuming that a initial known function $u_{0}(x, t)$ is given, we can obtain following formula after taking the $u_{0}(x, t)$ into the formula (4):

$$
\mathcal{L} u(x, t)+\mathcal{N} u_{0}(x, t)=f(x, t) .
$$


So, the equation (4) is transformed into a linear equation (5). We can obtain a new function $u_{1}(x, t)$ by solving euation (5), and get following linear iterative scheme:

$$
\mathcal{L} u_{n}(x, t)+\mathcal{N} u_{n-1}(x, t)=f(x, t) .
$$

If the linear iterative scheme (6) is convergent, then $u_{n}(x, t) \rightarrow u(x, t), n \rightarrow \infty$.

For a given control precision $\varepsilon$, if $\left|u_{n}(x, t)-u_{n-1}(x, t)\right| \leqslant \varepsilon$, the iteration stops. Thus we can get the numerical solution of the equation (4). This is the process of direct linearized iteration method.

By the above linear iterative process, we have transformed a nonlinear KdVB equation into a linear equation. Next, we introduce the the partial differential matrix of barycentric interpolation.

\subsection{The Partial Differential Matrix of BICM}

In the region $\Omega=[a, b] \times[0, T]$, we divide the interval $[a, b]$ into $M$ different nodes: $a=x_{1}<x_{2}<\cdots<x_{M}=b$. In the interval $[0, T]$, there is $N$ different nodes: $0=t_{1}<t_{2}<\cdots<t_{N}=T$. In the region $\Omega$, those nodes generate tensor type nodes, that is: $\left\{\left(x_{i}, t_{j}\right), i=1,2, \cdots, M ; j=1,2, \cdots, N\right\}$.

The value of function $u(x, t)$ at nodes $\left(x_{i}, t_{j}\right)$ is defined as:

$$
u_{i j}=u\left(x_{i}, t_{j}\right), i=1,2, \cdots, M, j=1,2, \cdots, N .
$$

The barycentric interpolation of $u(x, t)$ at nodes $\left(x_{i}, t_{j}\right)$ can be written as

$$
u(x, t)=\sum_{i=1}^{M} \sum_{j=1}^{N} \xi_{i}(x) \eta_{j}(t) u_{i j}, i=1,2, \cdots, M, j=1,2, \cdots, N .
$$

The $\frac{\partial^{l+k} u}{\partial x^{\prime} \partial t^{k}}(l+k$ order partial derivative of function $u(x, t))$ can be written as:

$$
\frac{\partial^{l+k} u}{\partial x^{l} \partial t^{k}}=\sum_{i=1}^{M} \sum_{j=1}^{N} \xi_{i}^{(l)}(x) \eta_{j}^{(k)}(t) u_{i j}, l, k=1,2, \cdots
$$

and the $l+k$ order partial derivatives of function $u(x, t)$ at nodes $\left(x_{p}, t_{q}\right)$ are defined as:

$$
u^{(l, k)}\left(x_{p}, t_{q}\right):=\frac{\partial^{l+k} u\left(x_{p}, t_{q}\right)}{\partial x^{l} \partial t^{k}}=\sum_{i=1}^{M} \sum_{j=1}^{N} \xi_{i}^{(l)}\left(x_{p}\right) \eta_{j}^{(k)}\left(t_{q}\right) u_{p q}, p=1,2, \cdots, M ; q=1,2, \cdots, N .
$$

Let $x^{0}=\left[x_{1}, x_{2}, \cdots, x_{M}\right]^{T}, t^{0}=\left[t_{1}, t_{2}, \cdots, t_{N}\right]^{T}$ be respectively defined as the column vectors of nodes at $x, t$ axis, and the matrixes $X, T$ composed of tensor type node coordinates are respectively defined as:

$$
X=\left[\begin{array}{c}
\left(x^{0}\right)^{T} \\
\left(x^{0}\right)^{T} \\
\vdots \\
\left(x^{0}\right)^{T}
\end{array}\right], T=\left[t^{0}, t^{0}, \cdots, t^{0}\right] .
$$

Stretching the matrix $X$ and $T$ into following $N \times M$ dimensional column vectors $x, t$ by columns:

$$
x=\left[X_{1}, X_{2}, \cdots, X_{M \times N}\right]^{T}, t=\left[T_{1}, T_{2}, \cdots, T_{M \times N}\right]^{T} .
$$

There is the following relationships between the components of vector $x, t$ and the components of vector $x^{0}, t^{0}$ :

$$
X_{k}=X_{(i-1) N+j}=x_{i}, T_{k}=T_{(i-1) N+j}=t_{j}, i=1,2, \cdots, M ; j=1,2, \cdots, N ; k=1,2, \cdots, M \times N .
$$

To facilitate, let

$u=\left[u_{1}, u_{2}, \cdots, u_{M \times N}\right]^{T}, u^{(l, k)}=\left[u_{1}^{(l, k)}, u_{2}^{(l, k)}, \cdots, u_{M \times N}^{(l, k)}\right]^{T}$,

$u_{p}=u\left(X_{p}, T_{p}\right), u_{p}^{(l, k)}=u^{(l, k)}\left(X_{p}, T_{p}\right), p=1,2, \cdots, M \times N$.

So, equation 13 can be written as follows:

$$
u^{(l, k)}=D^{(l, k)} u \text {. }
$$


where, $D^{(l, k)}=C^{(l)} \otimes D^{(k)}$ is the Kronecker product of matrix $C^{(l)}$ and $D^{(k)}$, and we call it as $l+k$ order partial differential matrix at nodes $\left\{\left(x_{i}, t_{j}\right), i=1,2, \cdots, M ; j=1,2, \cdots, N\right\} . C^{(l)}$ and $D^{(k)}$ are $l$ order differential matrix on $x$ direction nodes and $k$ order differential matrix on $t$ direction nodes. Denote:

$$
C^{(0)}=I_{M}, D^{(0)}=I_{N} .
$$

$I_{M}$ is $M$ order unit matrix and $I_{N}$ is $N$ order unit matrix.

\subsection{The BICM in the KdVB Equation}

Consider the KdVB equation(1), which satisfies following initial condition

$$
u(x, 0)=f(x), a<x<b, t>0,
$$

and boundary condition

$$
u(a, t)=f_{1}(t), u(b, t)=f_{2}(t), t>0 .
$$

Using the given initial hypothesis function $u_{0}(x, t)$, the equation(1) can be directly linearized to the following equation:

$$
\frac{\partial u}{\partial t}+\varepsilon u_{0} \frac{\partial u}{\partial x}-v \frac{\partial^{2} u}{\partial x^{2}}+\mu \frac{\partial^{3} u}{\partial x^{3}}=0, a<x<b, t>0,
$$

using formula (18), we can obtain following linear iterative format:

$$
\frac{\partial u_{n}}{\partial t}+\varepsilon u_{n-1} \frac{\partial u_{n}}{\partial x}-v \frac{\partial^{2} u_{n}}{\partial x^{2}}+\mu \frac{\partial^{3} u_{n}}{\partial x^{3}}=0, a<x<b, t>0,
$$

so, equation(1) can be written in following matrix form

$$
\mathbf{L} \mathbf{U}=\mathbf{0} .
$$

Where, $\mathbf{L}=D^{(0,1)}+\operatorname{cdiag}\left(u_{n-1}\right) D^{(1,0)}-v D^{(2,0)}+\mu D^{(3,0)}$, $\mathbf{U}=\left[u_{1}, u_{2}, \cdots, u_{n}\right]^{T}, \mathbf{0}=[0,0, \cdots, 0]$.

\subsection{Applying Method of Initial Boundary Conditions}

Use collocation method to solve the differential equation problems, the key is how to Handle the initial conditions. There are three methods. The first method is displacement method. The second method is supplemental method. The third method is elimination method.

In this paper, we use displacement method.

\section{Numerical Examples}

Now we apply the BICM to the KdVB equation. In order to verify the accuracy of the proposed BICM for the equation, we report the numerical error norms as measure of comparison. The discrete $L_{2}$ and $L_{\infty}$ error norms are defined as follows:

$$
\begin{aligned}
& L_{2}(u)=\left(\sum_{i=1}^{M}\left|u\left(x_{i}, t\right)-u_{n}\left(x_{i}, t\right)\right|^{2} \Delta x\right)^{\frac{1}{2}}, \\
& L_{\infty}(u)=\max _{1 \leq i \leq M}\left|u\left(x_{i}, t\right)-u_{n}\left(x_{i}, t\right)\right|,
\end{aligned}
$$

Here, $u\left(x_{i}, t\right)$ is exact solution and $u_{n}\left(x_{i}, t\right)$ is numerical solution.

Example 1 We consider the KdVB Eq.1 with $\varepsilon=1$

$$
u_{t}+u u_{x}-v u_{x x}+\mu u_{x x x}=0,0<x<10, t>0 .
$$

The exact solution is: $u(x, t)=-\frac{6 v^{2}}{25 \mu}\left(1+\tanh (\xi)-\frac{1}{2} \operatorname{sech}^{2}(\xi)\right)$, where, $\xi=\frac{v}{10 \mu}\left(x+\frac{6 v^{2}}{25 \mu}\right) t$.

In Figure 1, the comparisons between exact and numerical solutions of $u$ obtained by present method at $t=0.01$ are visualized. From Figure 1, it is observed that the numerical solution is almost equal to the exact solution. 


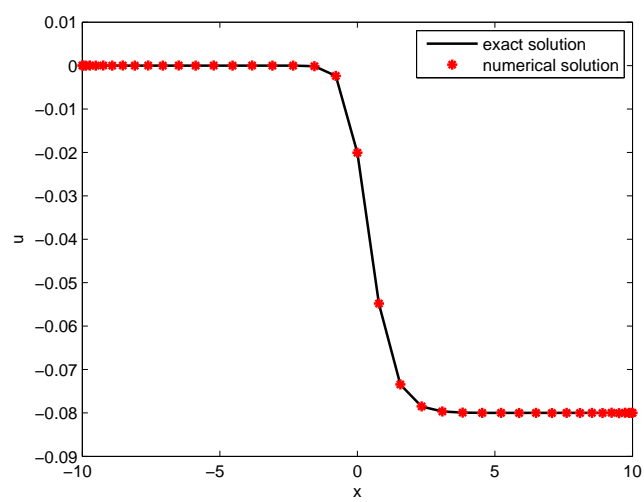

Figure 1. Comparisons between numerical and exact solutions obtained by present method for example 1 with $v=0.1, \mu=0.01, t=0.01, M=40, N=$ 20

Example 2 We consider the homogeneous Burgers' equation 2 with $\varepsilon=1$ :

$$
u_{t}+u u_{x}-v u_{x x}=0 . a<x<b, t>1
$$

The exact solution of this equation is:

$$
u(x, t)=\frac{x / t}{1+\sqrt{t / t_{0}} e^{x^{2} / 4 v t}}, t_{0}=e^{1 / 8 v}
$$

The initial and boundary conditions are determined by above exact solution.

$L_{2}$ and $L_{\infty}$ error norms are presented at times $t=2,3$ and 4 in Table 1 which is a comparison of numerical results of present method with other method in the literature 9. Computations are done with $N=200(t=2), N=300(t=3)$, $N=400(t=4)$ for the method in the literature, and $N=20(t=2), N=30(t=3), N=40(t=4)$ for present method. From the Table 1, we can see that our method has higher accuracy than the method in the literature.

Both numerical and exact solutions visualized at $t=2$ in Figure 1, from which it is seen that the numerical solution is almost equal to the exact solution.

\begin{tabular}{|c|c|c|c|c|c|c|c|c|}
\hline \multirow[t]{3}{*}{$t$} & \multicolumn{4}{|c|}{$v=0.5(0<x<10)$} & \multicolumn{4}{|c|}{$v=0.05(0<x<3)$} \\
\hline & \multicolumn{2}{|c|}{$\begin{array}{l}Q B G A[9] \\
M=1000\end{array}$} & \multicolumn{2}{|c|}{$\begin{array}{c}\text { Present method } \\
M=70\end{array}$} & \multicolumn{2}{|c|}{$\begin{array}{l}Q B G A[9] \\
M=280\end{array}$} & \multicolumn{2}{|c|}{$\begin{array}{c}\text { Present method } \\
M=80\end{array}$} \\
\hline & $L_{2}(u)$ & $L_{\infty}(u)$ & $L_{2}(u)$ & $L_{\infty}(u)$ & $L_{2}(u)$ & $L_{\infty}(u)$ & $L_{2}(u)$ & $L_{\infty}(u)$ \\
\hline 2 & $1.63 \mathrm{E}-06$ & $1.33 \mathrm{E}-06$ & $1.2347 \mathrm{E}-13$ & $3.7691 \mathrm{E}-13$ & $6.0 \mathrm{E}-07$ & $8.6 \mathrm{E}-07$ & $1.0532 \mathrm{E}-13$ & $4.1607 \mathrm{E}-13$ \\
\hline 3 & 7.7E-07 & $5.8 \mathrm{E}-07$ & $1.3851 \mathrm{E}-13$ & 3.7735E-13 & $3.2 \mathrm{E}-07$ & $6.2 \mathrm{E}-07$ & $9.8720 \mathrm{E}-14$ & $3.8270 \mathrm{E}-13$ \\
\hline 4 & $2.45 \mathrm{E}-06$ & $5.28 \mathrm{E}-06$ & $2.7208 \mathrm{E}-13$ & $4.1601 \mathrm{E}-13$ & 4.61E-06 & $1.70 \mathrm{E}-05$ & $1.7748 \mathrm{E}-13$ & $4.5544 \mathrm{E}-13$ \\
\hline
\end{tabular}

Table 1. Comparison of error norms for the various values of $t$ for Example 2

Example 3 We consider the following nonhomogeneous Burgers' equation:

$$
u_{t}+u u_{x}-v u_{x x}=-2 x\left(e^{-x^{2}-t}\right)^{2}+\left(2 v-1-4 v x^{2}\right)\left(e^{-x^{2}-t}\right),-5<x<5, t>0 .
$$

The exact solution is: $u(x, t)=e^{-x^{2}-t}$.

The $L_{2}$ error norms for different values of $M, N$ with $v=0.2, t=1$ are shown in Table 3 . The minimum error norm can reach $O\left(10^{-14}\right)$. The absolute error of $u(x, t)$ obtained by present method is shown in Figure 2. 


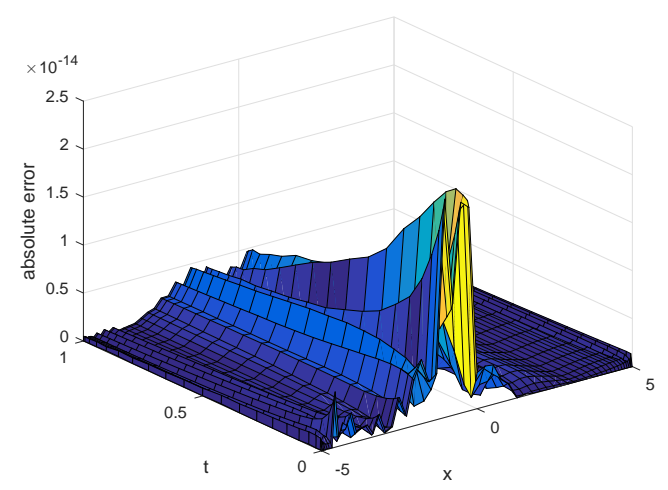

Figure 3. The absolute errors of $u$ obtained by present method for example 3 with $v=0.2, t=$ $1, M=60, N=20$.

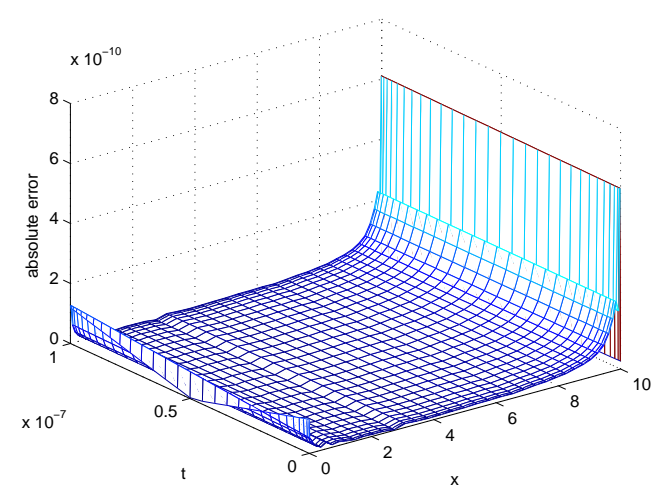

Figure 4. The absolute error of $u$ obtained by present method for example 4 with $M=40, N=30$, $t=10^{-7}$.

Table 2. Comparison of $L_{2}(u)$ error norm for Example 3 with $v=0.2, t=1.0$.

\begin{tabular}{ccccccccc}
\hline$M$ & $N$ & NFDC [10] & Present method & & $M$ & $N$ & NFDC [10] & Present method \\
\hline 56 & 10 & $1.0020 \mathrm{E}-02$ & $9.1354 \mathrm{E}-13$ & & 50 & 9 & $1.2576 \mathrm{E}-02$ & $6.8318 \mathrm{E}-11$ \\
95 & 20 & $3.4974 \mathrm{E}-03$ & $1.1272 \mathrm{E}-12$ & & 100 & 22 & $3.1614 \mathrm{E}-03$ & $1.1914 \mathrm{E}-12$ \\
159 & 40 & $1.2524 \mathrm{E}-03$ & $2.3775 \mathrm{E}-12$ & & 200 & 54 & $7.9231 \mathrm{E}-04$ & $3.2237 \mathrm{E}-12$ \\
267 & 80 & $4.4534 \mathrm{E}-04$ & $1.0053 \mathrm{E}-11$ & & 400 & 137 & $1.9882 \mathrm{E}-04$ & $8.8320 \mathrm{E}-14$ \\
\hline
\end{tabular}

Example 4 We consider the KdV Eq.3 with $\varepsilon=-6, \mu=1$ :

$$
u_{t}-6 u u_{x}+u_{x x x}=0
$$

Where $0<x<10, t>0$.

Eq.27 satisfy the initial condition

$$
u(x, 0)=-2 \operatorname{sech}^{2}(x), 0<x<10 .
$$

and the boundary conditions

$$
u(0, t)=-2 \operatorname{sech}^{2}(-4 t), u(10, t)=-2 \operatorname{sech}^{2}(10-4 t), t>0 .
$$

The exact solution is: $u(x, t)=-\operatorname{sech}^{2}(x-4 t)$.

The Table 3 give the exact solution, numerical solution, and absolute error for the various values of $t$ with $M=40$, $N=30$. And the absolute error at $t=10^{-7}$ was visualized in Figure 1 .

Table 3. Comparison of numerical results for Example 4 with $M=40, N=30$.

\begin{tabular}{cccc}
\hline$t$ & $u(x, t)$ & $u_{n}(x, t)$ & $\left|u(x, t)-u_{n}(x, t)\right|$ \\
\hline $1 \mathrm{E}-1$ & $-3.6697 \mathrm{E}-08$ & $-1.3883 \mathrm{E}-05$ & $1.38461 \mathrm{E}-05$ \\
$1 \mathrm{E}-2$ & $-1.7863 \mathrm{E}-08$ & $1.9923 \mathrm{E}-08$ & $3.77855 \mathrm{E}-08$ \\
$1 \mathrm{E}-3$ & $-1.6622 \mathrm{E}-08$ & $-6.1112 \mathrm{E}-08$ & $4.44899 \mathrm{E}-08$ \\
$1 \mathrm{E}-4$ & $-1.6502 \mathrm{E}-08$ & $-1.5483 \mathrm{E}-08$ & $1.01893 \mathrm{E}-09$ \\
$1 \mathrm{E}-5$ & $-1.6491 \mathrm{E}-08$ & $-1.6394 \mathrm{E}-08$ & $9.66932 \mathrm{E}-11$ \\
$1 \mathrm{E}-6$ & $-1.6489 \mathrm{E}-08$ & $-1.6460 \mathrm{E}-08$ & $2.98482 \mathrm{E}-11$ \\
$1 \mathrm{E}-7$ & $-1.6489 \mathrm{E}-08$ & $-1.6505 \mathrm{E}-08$ & $1.56360 \mathrm{E}-11$ \\
\hline
\end{tabular}

\section{Discussion}

In this paper, the nonlinear KdVB equations have solved by using barycentric interpolation collocation method (BICM). Numerical results on several examples indicate that the present method is better than some other methods available in the literature. There are many nonlinear problems to be solved to develop this method, We will study 
these problems in further work.

All programs of numerical examples are run by the MatlabR2013a and MatlabR2015b software.

\section{Acknowledgements}

We thank the unknown referees for their careful reading and helpful comments. This paper is supported by the National Natural Science Foundation of China (No.11361037), the Natural Science Foundation of Inner Mongolia(No.2015MS0118, No.2017MS0103), Numerical analysis of graduate course construction project of Inner Mongolia University of Technology(KC2014001).

\section{References}

Feng, P. H., Zhang, J. Z., Cao, S. L., \& Prants, S. V. (2017). Thermalized solution of the Galerkin-truncated Burgers equation: From the birth of local structures to thermalization. Communications in Nonlinear Science and Numerical Simulation, 45(2017), 104-116.

Hattam, L. (2017). KdV cnoidal waves in a traffic flow model with periodic boundaries. Journal of Physics D, 348(2017), 44-53.

Giuliani, F. (2017). Quasi-periodic solutions for quasi-linear generalized KdV equations. Journal of Differential Equations, 262(2017), 5052-5132.

Mukundan, V., \& Awasthi, A. (2015). Efficient numerical techniques for Burgers equation. Applied Mathematics and Computation, 262 (2015), 282-297.

Jiwari, R. (2015). A hybrid numerical scheme for the numerical solution of the Burgers equation. Computer Physics Communications, 188 (2015), 59-67.

Jiwari, R., Mittal, R. C., \& Sharma, K. K. (2013). A numerical scheme based on weighted average differential quadrature method for the numerical solution of Burgers equation. Applied Mathematics and Computation, 219(2013), 6680-6691.

Su, C.H., \& Gardner, C.S. (1969). Derivation of the Korteweg-de Vries and Burgers equation. Journal of Mathematical Physics, 10(1969), 536-539.

Seadawya, A. R. (2017). The generalized nonlinear higher order of KdV equations from the higher order nonlinear Schrödinger equation and its solutions. Optik, 139(2017), 31-43.

Saka, B., \& DaĞ, I. (2013). Quartic B-spline Galerkin approach to the numerical solution of the KdVB equation. Applied Mathematics and Computation, 219(2013), 6680-6691.

Zheng, Q., Zhao, X., \& Liu Y. F. (2017). A novel finite difference scheme for Burgers' equation onunbounded domains. Applied Numerical Mathematics, 111(2017), 1-16.

\section{Copyrights}

Copyright for this article is retained by the author(s), with first publication rights granted to the journal.

This is an open-access article distributed under the terms and conditions of the Creative Commons Attribution license (http://creativecommons.org/licenses/by/3.0/). 